\title{
Intestinal obstruction and bacteremia - complications of capsule endoscopy
}

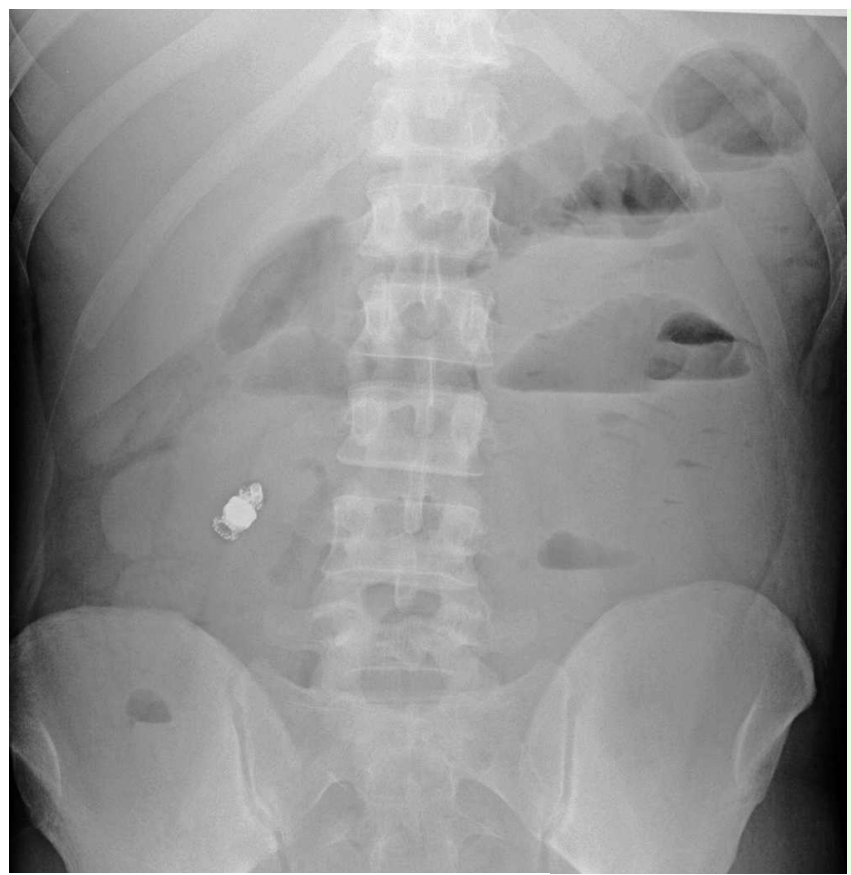

Fig. 1 Radiograph showing mechanical ileus.

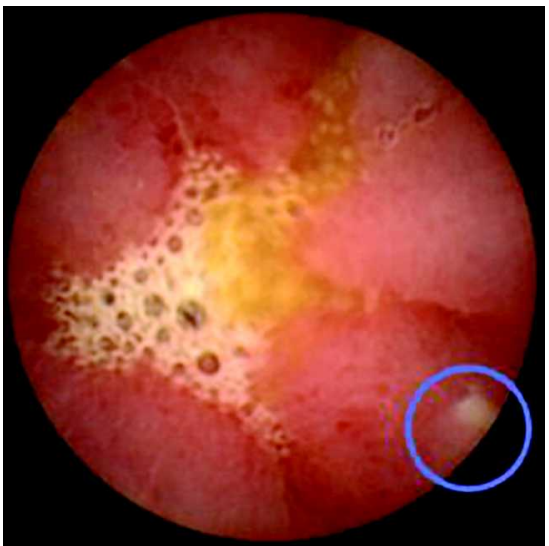

Fig. 2 Redness, swelling, and aphthae in the jejunum.

Capsule endoscopy is a modality for the evaluation of small bowel mucosa and has a high diagnostic yield for evaluation of suspected small intestinal Crohn's disease [1]. Capsule endoscopy is superior to barium follow-through in diagnosing patients with Crohn's disease [2,3]. The most serious complication of capsule endoscopy is mechanical obstruction caused by capsule impaction in a stricture. We present a case of mechanical ileus and bacteremia caused by impaction of the capsule in a patient with suspected Crohn's disease, but with a normal

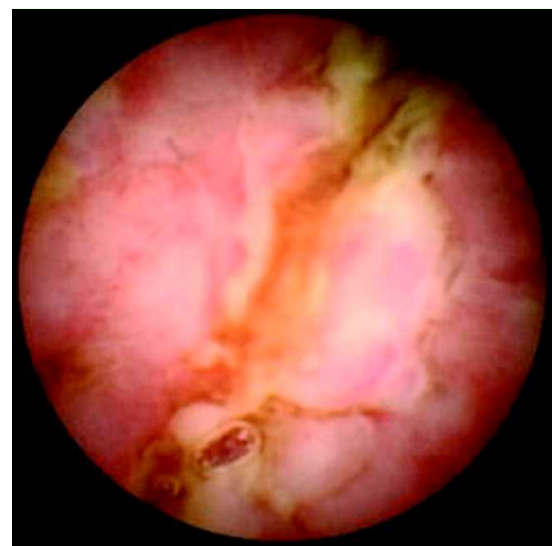

Fig. 3 Stenosis and ulceration.

barium follow-through prior to the endoscopy.

The patient was admitted to our hospital for capsule endoscopy due to weight loss, diarrhea, and abdominal pain. Prior to the procedure, a barium follow-through and ileocolonoscopy were carried out and revealed no abnormalities. The patient experienced abdominal pain and vomiting 5 hours after capsule ingestion. Abdominal radiograph was normal. The following day, temperature reached $41.5^{\circ} \mathrm{C}$ and a radiograph showed small intestinal obstruction ( Fig. 1). Capsule endoscopy

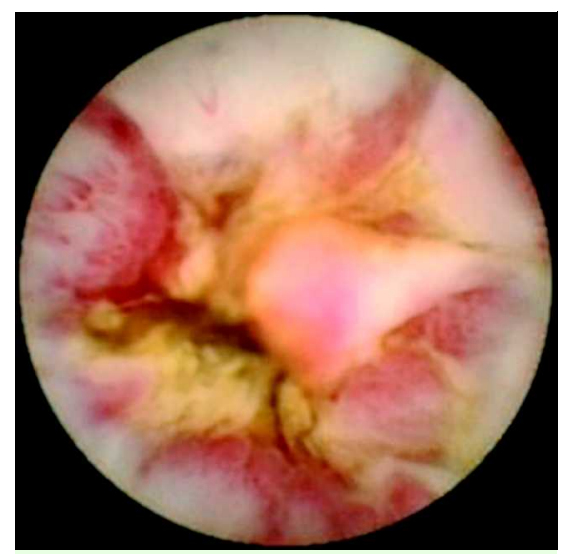

Fig. 4 Stenosis caused by ulceration.

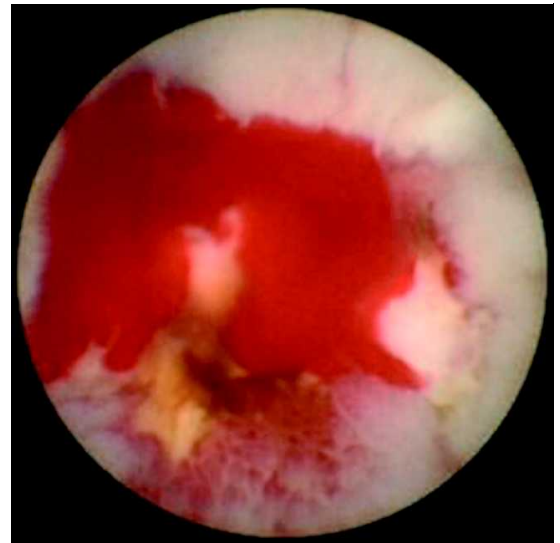

Fig. 5 Bleeding caused by capsule progression through stenotic area.

revealed ulcerations and stenosis compatible with Crohn's disease ( Fig. 2-5). The patient was treated with intravenous antibiotics and prednisolone. Abdominal radiograph was normal 2 days later. Blood culture was positive for Clostridium perfringens. A week after the procedure, the capsule had passed, and the patient was discharged with no abdominal symptoms and with tapering oral steroid dosage.

This case demonstrates an acute, rare, and serious complication of capsule endoscopy, and confirms that small-bowel follow-through does not reliably detect an intestinal stenosis, which should be suspected in patients with abdominal pain related to meals, and an involuntary weight loss. The risk of capsule retention is estimated to be $1.4 \%$ in patients with suspected Crohn's disease, and $13 \%$ in patients with Crohn's disease [4,5]. In the present case, the standard diagnostic approach did not reveal the cause of symptoms, whereas capsule endoscopy enabled treatment and a positive therapeutic outcome. 
Endoscopy_UCTN_Code_CPL_1AI_2AB

G. Dam, M. J. Nielsen, L. A. Christensen Aarhus University Hospital, Medical Department V, Denmark

\section{References}

1 Triester SL, Leighton JA, Leontiadis GI et al. A metaanalysis of the yield of capsule endoscopy compared to other diagnostic modalities in patients with non-stricturing small bowel Crohn's disease. Am J Gastroenterol 2006; 101: 954-964

2 Eliakim R, Fischer D, Suissa A et al. Wireless capsule video endoscopy is a superior diagnostic tool in comparison to barium followthrough and computerized tomography in patients with suspected Crohn's disease. Eur J Gastroenterol Hepatol 2003; 15: 363 367

3 Hara A, Leighton J, Height $R$ et al. Crohn disease of the small bowel. Radiology 2006; 238: $128-134$

4 Cave D, Legnani P, de Francis R et al. Consensus for capsule retention. Endoscopy 2005; 37: 1065 - 1067

5 Cheifetz AS, Kornbluth AA, Legnani $P$ et al. The risk of retention of the capsule endoscope in patients with known or suspected Crohn's disease. Am J Gastroenterol 2006; 101: $2218-2222$
Bibliography

DOI 10.1055/s-2007-995326

Endoscopy 2007; 39: E155-E156

(c) Georg Thieme Verlag KG Stuttgart · New York . ISSN 0013-726X

\section{Corresponding author}

\section{G. A. Dam, MD}

PET-Centret

Aarhus Univeristy Hospital

Nørrebrogade 44

8000 Aarhus $C$

Denmark

Fax: +45-8949-3020

gitte_dam@hotmail.com 